\title{
A Multi-Frequency VLBI Total Intensity and Polarization Study of the BL Lacertae Object $0716+714$
}

\author{
Y. Y. Kovalev \& D. C. Gabuzda \\ Astro Space Center of the Lebedev Physical Institute, Moscow, Russia \\ T. P. Krichbaum, W. Alef, \& A. Witzel \\ Max-Planck-Institut für Radioastronomie, Bonn, Germany
}

\begin{abstract}
New polarization-sensitive global VLBI and VLBA images of the BL Lacertae object $0716+714$ at $6 \mathrm{~cm}(1991.4)$ and $3.6 \mathrm{~cm} / 1.3 \mathrm{~cm}(1994.2)$ are presented. The source shows a compact, one-sided core-jet structure in structural position angle $\sim 13^{\circ}$. The $3.6 \mathrm{~cm}$ total intensity image shows the jet beginning to curve toward the arcsecond-scale structure, which lies in position angle $\sim-45^{\circ}$. Comparison with previously published data suggests that the apparent velocity of a $6 \mathrm{~cm}$ jet component is $>1.09 \pm 0.15 \mathrm{c}$, if the red shift of $0716+714$ is $z>0.3$. Linear polarization from the VLBI core was detected at all three frequencies; in addition, polarization from the innermost jet component was detected at $1.3 \mathrm{~cm}$. This compact jet polarization appears to be quite high, $\sim 50 \%$, and to be aligned with the jet direction, which can be understood if it is a transverse shock. The VLBI core polarization at our earlier epoch was perpendicular to the VLBI jet direction, but had rotated to align with the jet direction by the later epoch, about a year later. This rotation may indicate the imminent birth of a new VLBI component (shock).
\end{abstract}

\section{Introduction}

The intraday variable BL Lacertae object $0716+714$ has been intensively studied at a wide range of wavelengths (see Wagner et al. 1996; and refs. therein). The nature of the intraday variability in this source is not clear, though most investigators probably believe it to be intrinsic to the source. Previous VLBI observations by Eckart et al. (1987) revealed very compact structure, with a jet oriented nearly due North. Here, we present the first VLBI polarization observations of this source: a first epoch at $6 \mathrm{~cm}$ and a second epoch at $3.6 / 1.3 \mathrm{~cm}$.

\section{Observations and Reduction}

Our first $(6 \mathrm{~cm})$ observing epoch was 1991.43, with a global array of ten antennas (Onsala, Medicina, Bonn, Jodrell Bank, Haystack, Green Bank, Los Alamos$V_{L B A}{ }^{1}$, VLA, Pie Town-VLBA, Owens Valley-VLBA); these data were correlated at Haystack. Our second $(3.6 / 1.3 \mathrm{~cm})$ observing epoch was in 1994.22 , using the ten VLBA antennas; these data were correlated at Bonn. Hybrid maps of the distribution of total intensity $I$ and the associated linear polarization $P$ were made in both AIPS and the Brandeis VLBI package. Model fits to the calibrated $I$ and $P$ visibilities were then obtained in the Brandeis package. The images obtained in the two packages were very similar.

\footnotetext{
${ }^{1}$ The National Radio Astronomy Observatory is a facility of the National Science Foundation, operated under a cooperative agreement by Associated Universities, Inc.
} 


\section{Results and Discussion}

The VLBI jet in structural position angle $\sim 13^{\circ}$ is clearly visible in all our images; the $3.6 \mathrm{~cm}$ image shows the jet beginning to curve toward the arcsecond-scale structure in position angle $\sim-45^{\circ}$. The $6 \mathrm{~cm}$ VLBI structure at epoch 1991.43 is dominated by a core plus two jet components $\sim 0.77(\mathrm{C} 2)$ and 2.08 (C3) mas from the core. Eckart et al. (1987) observed an expanding double structure in $0716+714$ at $6 \mathrm{~cm}$ at epochs 1979.93 and 1983.25 . The predicted location for their jet component at our $6 \mathrm{~cm}$ epoch (assuming that it continued to travel outward from the core at the speed suggested by their two epochs) is $\sim 2.3$ mas, near the separation of C3, $r=2.08$ mas. If this is the same component, it moved with a nearly constant speed $0.07 \pm 0.01 \mathrm{mas} / \mathrm{yr}$ over a period of fifteen years or more. We also detected this component at $3.6 \mathrm{~cm}$ at epoch 1994.22 , at a separation of $r=2.31$. There is no obvious discrepancy in the position of the $3.6 \mathrm{~cm}$ component and the expected position of $\mathrm{C} 3$ based on the proper motion inferred by the three $6 \mathrm{~cm}$ epochs. The apparent velocity is $>1.09 \pm 0.15 \mathrm{c}$ $\left(H_{0}=75 \mathrm{~km} \mathrm{~s}^{-1} \mathrm{Mpc}^{-1}, q_{0}=0.5\right.$ ), if the red shift $z>0.3$ (Wagner et al. 1996). This is consistent with the evidence that the superluminal speeds in BL Lacertae objects are on average lower than those in quasars (Gabuzda et al. 1994).

The spectral index of the core $\alpha_{8.4-22.2}=-0.1\left(F_{\nu} \sim \nu^{\alpha}\right)$, indicating that it may be optically thick, as expected. The spectral indices for the innermost three knots in the VLBI jet are $-0.9,-0.33$, and -0.41 . When we have identified components in the 3.6 and $1.3 \mathrm{~cm}$ images, their positions at the two wavelengths have agreed to within the estimated errors (except for the innermost knot, whose position at $3.6 \mathrm{~cm}$ is poorly determined, since it is barely resolved from the core); we therefore did not try to take account of possible frequency-dependent shifts in the VLBI core positions (Lobanov 1996), since it appears that these effects are too small to be visible in our data.

The degree of linear polarization for the VLBI core is $2-4 \%$, as is typical for BL Lacertae objects (Gabuzda et al. 1994). The core polarization at our earlier epoch was perpendicular to the jet direction, but had rotated to align with the jet direction by the later epoch. This rotation may indicate the imminent birth of a new VLBI component (shock), as was the case for OJ 287 (Gabuzda, Wardle, \& Roberts 1989) and 0454+844 (Gabuzda et al. 1994).

Linear polarization was detected from the innermost jet component at $1.3 \mathrm{~cm}$ at epoch 1994.22 . The polarization is quite strong, $\sim 47 \pm 17 \%$, and is aligned with the jet direction, as would be expected if it were associated with a transverse shock (the inferred magnetic field is perpendicular to the jet). This high degree of polarization leaves no doubt that this emission is optically thin, and that the magnetic field is rather well ordered.

\section{References}

Eckart, A., et al. 1987. $A \mathscr{E} A S, 67,121-146$.

Gabuzda, D. C., Wardle, J. F. C., \& Roberts, D. H. 1989. ApJ, 336, L59-62.

Gabuzda, D. C., et al. 1994. ApJ, 435, 140-161.

Lobanov, A. P. 1996. Ph.D. Thesis, New Mexico Institute of Mining \& Technology.

Wagner, S. J., et al. 1996. $A J, 111,2187-2211$. 